\title{
On the Generation of Electronic Ring Currents under Vibronic Coupling Effects
}

\author{
Krishna Reddy Nandipati ${ }^{1, \text { a) }}$ and Oriol Vendrell ${ }^{1,2, \text { b) }}$ \\ 1) Theoretische Chemie, Physikalisch-Chemisches Institut, Universität Heidelberg, Im Neuneheimer Feld 229, \\ 69120 Heidelberg, Germany \\ ${ }^{2)}$ Centre for Advanced Materials, Universität Heidelberg, Im Neuenheimer Feld 205, 69120 Heidelberg, \\ Germany
}

(Dated: 7 October 2020)

We study the generation of electronic ring currents in the presence of nonadiabatic coupling using circularly polarized light. For this, we introduce a solvable model consisting of an electron and a nucleus rotating around a common center and subject to their mutual Coulomb interaction. The simplicity of the model brings to the forefront the non-trivial properties of electronic ring currents in the presence of coupling to the nuclear coordinates and enables the characterization of various limiting situations transparently. Employing this model, we show that vibronic coupling effects play a crucial role even when a single $E$ degenerate eigenstate of the system supports the current. The maximum current of a degenerate eigenstate depends on the strength of the nonadiabatic interactions. In the limit of large nuclear to electronic masses, in which the Born-Oppenheimer approximation becomes exact, constant ring currents and time-averaged oscillatory currents necessarily vanish.

\section{INTRODUCTION}

Electronic currents triggered by circularly polarized light in ring-shaped molecules ${ }^{1-10}$ and materials ${ }^{11-13}$ have become a subject of much interest due to their potential applications in optoelectronics ${ }^{14}$, e.g. as a platform for fast switching qubits ${ }^{13}$. Recent advances in femto- and attosecond laser technology ${ }^{15,16}$ have motivated theoretical investigations on the generation of electronic ring currents in time-scales comparable to the fastest vibrational dynamics in molecules ${ }^{9,10,17}$. It is now well established that ultrashort laser pulses with a sufficient bandwidth can trigger the migration of electronic charge across molecular structures in attosecond timescales $^{18-21}$. Ultrafast charge migration implies the superposition of electronic states with relatively large energy gaps between them, which often feature very different potential energy surfaces. Hence, after the electrons are set in motion, nuclei quickly respond to the electronic excitation and inevitably lead to decoherence ${ }^{22,23}$ and electronic energy relaxation through vibronic coupling effects $^{24,25}$. On the other hand, circular ring currents require the presence of doubly degenerate $E$ electronic components, present in molecular systems with at least a 3-fold or higher symmetry axis, and one may be lead to believe that the coupling between electrons and nuclei and the corresponding vibronic coupling effects will not substantially affect the laser-triggered electronic circulation in these systems.

Up to now, most theoretical studies on the generation and control of ring currents by applying circularly polarized laser fields have not considered vibronic coupling effects ${ }^{1-9,11-13}$. The relevant time-scales for these

\footnotetext{
a) e-mail: krishna.nandipati@pci.uni-heidelberg.de

b) e-mail: oriol.vendrell@pci.uni-heidelberg.de
}

currents are at least tens of femtoseconds. Therefore, it is inevitable that vibronic effects will influence to some extent the electronic dynamics ${ }^{24,25}$ : it has been reported that the build up of strong correlations between electrons and nuclei can take as little as 1 to 2 fs in the vicinity of a conical intersection ${ }^{26}$. Previous studies by Kanno et. al. considering vibronic effects on electronic currents focused on systems without actual state degeneracy and instead described currents triggered by linearly polarized light in chiral systems ${ }^{10,27,28}$. In these systems, the currents achieved are oscillatory because of the different energy of the eigenstates involved.

The purpose of the present work is to unravel the specific role of vibronic coupling effects in the generation of sustained ring currents by circularly polarized light in systems with two degenerate circulation directions. Owing to their stationary nature, constant currents must be necessarily supported by a very small number of energy eigenstates of the complete molecular Hamiltonian, even only one, which belong to degenerate pairs. Even in cases where only one eigenstate is involved, we will discuss how vibronic effects can be very significant and can lead to an almost complete suppression of the ring current. We will also establish the general requirements and laser pulse properties needed in order to achieve either stable and sustained currents, or highly oscillatory ones. To this end, we consider a model system consisting of two concentric rings, the innermost one constraining the motion of a positively charged atomic nucleus, and the outermost one constraining the motion of an electron around a common center. The two rings lie on the $(x, y)$-plane and the laser field is assumed to propagate perpendicular to the plane of the particles along the $z$-direction. Despite its simplicity, this ring-ring-model (RRM) fulfills two key characteristics: first, it captures the fundamental features of molecular systems that are able to present circular electronic currents with two de- 
generate rotation directions and second, it captures the effect of the coupling to the nuclei. On the one hand, the RRM consists of a rotational axis of at least third order, resulting in doubly degenerate vibronic states of an $E$ symmetry representation within the corresponding point group. On the other hand, it features a nuclear coordinate that breaks this symmetry for the electronic subsystem if fixed at an arbitrary point in nuclear configuration space. The model represents a class of vibronic coupling phenomena resulting from either Renner-Teller $(\mathrm{RT})^{29}$ or Jahn-Teller $(\mathrm{JT})^{30}$ effects, in which the nuclear motion of appropriate symmetry couples the doubly degenerate electronic states of $E$ symmetry. The RRM including an external laser field is considered numerically exactly without invoking the BO approximation. Moreover, several of its important features can be understood based on analytical considerations. We also show how the Hamiltonian of the RRM can be expressed as a vibronic coupling Hamiltonian $(\mathrm{VCH})^{31}$ in a diabatic electronic basis, which is an exact representation as well. This fact underpins an important observation, namely that a $\mathrm{VCH}$ provides the necessary theoretical framework to describe electronic ring currents under nonadiabatic couplings between electrons and nuclei.

\section{THEORY}

In the following we introduce the RRM system and some theoretical considerations. The electronic and nuclear motion are constrained to two concentric rings of fixed radii $r$ and $R$, respectively, lying on the $(x, y)$-plane. The electronic and nuclear angular degrees of freedom are denoted by $\theta$ and $\alpha$, respectively. The total Hamiltonian including the light-matter interaction term within the semiclassical dipole-approximation ${ }^{32}$ reads

$$
\begin{aligned}
\hat{H} & =\hat{H}_{\mathrm{mol}}-\boldsymbol{\mu} \cdot \mathbf{E}(t) \\
& =\hat{T}_{\alpha}+\hat{T}_{\theta}+\hat{V}(\alpha, \theta)-\boldsymbol{\mu} \cdot \mathbf{E}(t)
\end{aligned}
$$

with kinetic energy (KE) terms

$$
\hat{T}_{\gamma}=-\frac{\hbar^{2}}{2 I_{\gamma}} \frac{\partial^{2}}{\partial \gamma^{2}} \quad \gamma \rightarrow(\alpha, \theta)
$$

and moment of inertia $I_{\gamma}=m_{\gamma} r_{\gamma}^{2}$ with $r_{\gamma} \rightarrow(R, r)$ and $m_{\gamma} \rightarrow(M, m)$. Here $M$ and $m$ are the mass of the nucleus and electron, respectively. The Coulomb interaction of the electron and the nucleus is then given by

$$
\begin{aligned}
\hat{V}(\alpha, \theta) & =\frac{-Q e^{2}}{\sqrt{(r \cos \theta-R \cos \alpha)^{2}+(r \sin \theta-R \sin \alpha)^{2}}} \\
& =\frac{-Q e^{2}}{\sqrt{\left(r^{2}+R^{2}-2 r R \cos (\theta-\alpha)\right.}} \\
& =\frac{-Q e^{2}}{\sqrt{(A-B \cos (\theta-\alpha)}},
\end{aligned}
$$

where $Q$ is the charge of the nucleus and the $A$ and $B$ constants are implicitly defined. Although the $2 \mathrm{D}$ Coulomb interaction can be handled numerically in the solution of the time-dependent Schrödinger equation (TDSE), we introduce here a second order approximation to the interaction similar in spirit to a multipolar expansion, namely the potential is expanded to second order around $\cos (\theta-\alpha)=0$. This approximation is very good as long as the radius of the outer ring is several times larger than the inner ring, as in our case, and leads to the interaction potential

$$
\hat{V}(\alpha, \theta)=v_{0}-\frac{1}{2} \kappa_{1} \cos (\theta-\alpha)-\frac{1}{4} \kappa_{2} \cos ^{2}(\theta-\alpha) .
$$

with $v_{0}=-Q e^{2} / \sqrt{A}, \kappa_{1}=Q e^{2} B / A^{3 / 2}$ and $\kappa_{2}=$ $3 Q e^{2} B^{2} / 2 A^{5 / 2}$. The first term $v_{0}$ is the constant chargecharge interaction, which is subtracted from the potential in the following. The term proportional to $\cos (\theta-\alpha)$ couples the doubly degenerate electronic states with the nondegenerate ground electronic state, whereas the term proportional to $\cos ^{2}(\theta-\alpha)$ couples directly the doubly degenerate electronic states. This expansion of the Coulomb interaction facilitates the analytical description of the electron-nucleus interaction terms and of the angular momentum transfer among the two subsystems. In the numerical simulations of the RRM system the parameters are $(r=20, R=1, m=1, M=2000, Q=1)$, all in atomic units, unless otherwise specified.

We introduce now a Born-Huang $(\mathrm{BH})$ expansion $^{33}$ of the nuclear-electronic wave function $\psi(\alpha, \theta)$ as is customary in the description of nonadiabatic effects in molecular systems. A convenient diabatic electronic basis are the eigenstates of the uncoupled electron, $\hat{T}_{\theta}$, given by $\varphi_{l}(\theta)=e^{i l \theta} / \sqrt{2 \pi}$, resulting in the wave function

$$
\psi(\alpha, \theta, t)=\sum_{l=-L}^{L} \chi_{l}(\alpha, t) \varphi_{l}(\theta)=\sum_{l=-L}^{L} \chi_{l}(\alpha, t)\left\{\frac{1}{\sqrt{2 \pi}} e^{i l \theta}\right\},
$$

where the population of each electronic state follows from the norm-squared of the nuclear amplitude $P_{l}(t)=$ $\left\langle\chi_{l}(t) \mid \chi_{l}(t)\right\rangle$. In the discussion below it will be sometimes useful to expand the nuclear wavepackets in the basis of eigenstates of the uncoupled nuclear coordinate, $\chi_{l}(\alpha, t)=\frac{1}{\sqrt{2 \pi}} \sum_{k=-\nu}^{\nu} a_{k}^{l}(t) e^{i k \alpha}$. The matrix representation of the vibronic coupling Hamiltonian $(\mathrm{VCH})$ with potential matrix elements $\left\langle\varphi_{l^{\prime}}\left|\left(\hat{H}_{\mathrm{mol}}-\hat{T}_{\alpha}\right)\right| \varphi_{l}\right\rangle$ for $L=1$, i.e. in the space of electronic eigenstates with quantum numbers $l=\{-1,0,1\}$, reads

$$
\boldsymbol{H}=\hat{T}_{\alpha} \boldsymbol{I}_{3 \times 3}+\left(\begin{array}{ccc}
\frac{\hbar^{2}}{2 I_{\theta}} & \frac{\kappa_{1}}{4} e^{i \alpha} & \frac{\kappa_{2}}{16} e^{2 i \alpha} \\
\frac{\kappa_{1}}{4} e^{-i \alpha} & 0 & \frac{\kappa_{1}}{4} e^{i \alpha} \\
\frac{\kappa_{2}}{16} e^{-2 i \alpha} & \frac{\kappa_{1}}{4} e^{-i \alpha} & \frac{\hbar^{2}}{2 I_{\theta}}
\end{array}\right)
$$

where $\boldsymbol{I}_{3 \times 3}$ is the $3 \times 3$ unit matrix. The resulting coupling scheme in the VCH follows transparently. The diabatic electronic state with $l=0$ couples with the $l=1$ and $l=-1$ states in first order, and this interaction 
changes the angular momentum of the nuclear coordinate $\alpha$ by $\pm \hbar$. On the other hand, the $l=+1$ and $l=-1$ states are directly coupled among each other in second order which changes the angular momentum of the nuclear coordinate $\alpha$ by $\pm 2 \hbar$. The present model is similar in spirit to that considered by Longuet-Higgins ${ }^{30}$ except for the fact that our model has an interaction potential with a periodicity of $2 \pi$ from the perspective of the electronic coordinate, whereas that of Longuet-Higgins has a periodicity of $\pi$, namely it is proportional to $\cos (2 \theta-\alpha)$. The $\pi$ periodicity of the Longuet-Higgins model results in the direct interaction between the degenerate electronic $E$ components already in first order, which corresponds to JT-type coupling ${ }^{30}$. In the taxonomy of JT and RT models, the RRM resembles a RT Hamiltonian, characterized by $2^{\text {nd }}$-order coupling terms, featuring both direct and indirect coupling of the degenerate electronic components. This fact makes it amenable to comparisons with general vibronic coupling situations in molecules.

The VCH in Eq. (6) provides a complete representation of the electronic-nuclear coupling of the system within the selected group of electronic states and under the coupling potential in Eq. (4). It has a similar structure compared to a molecular $\mathrm{VCH}$ with a $C_{n}$ symmetry axis of at least order $n=3$, where always doubly degenerate states of an $E$ symmetry representation are present ${ }^{34}$. Only the nuclear dependency of the coupling terms has a simpler structure than in molecular cases, where for example other coupling and tuning nuclear coordinates would be present $^{31}$. In the same way as the real-valued $p_{x}$ and $p_{y}$ orbitals of an atom, the real $\varphi_{x}^{E}$ and $\varphi_{y}^{E}$ electronic states of a molecular system can be combined to yield electronic states $\varphi_{ \pm}^{E} \propto \varphi_{x}^{E} \pm i \varphi_{y}^{E}$, featuring a net current; these are analogous to the $l=+1$ and $l=-1$ diabatic electronic states of the RRM model.

It is convenient in the following to introduce as a basis the product of electronic and nuclear angular momentum eigenstates $|k, l\rangle\left(\right.$ with $\left.\langle\alpha, \theta \mid k, l\rangle=\frac{1}{2 \pi} e^{i k \alpha} e^{i l \theta}\right)$, where $k$ and $l$ are the angular momentum quantum numbers of the electronic and nuclear degrees of freedom, respectively. The coupling between pairs of $|k, l\rangle$ basis states follows from simple integration of these with the interaction potential in Eq. (4):

$$
\left\langle k^{\prime}, l^{\prime}\left|\hat{V}^{(m)}(\alpha, \theta)\right| k, l\right\rangle=\beta_{m}\left(1-S\left(k-k^{\prime}\right) S\left(l-l^{\prime}\right)\right) \delta_{\left|k-k^{\prime}\right|, m} \delta_{\left|l-l^{\prime}\right|, m},
$$

where $m=1,2$ and $\beta_{1}=\kappa_{1} / 4 ; \beta_{2}=\kappa_{2} / 16$ and the function $S(z)$ returns the sign of its real argument modulo one. The presence of the sign functions $S\left(k-k^{\prime}\right)$ and $S\left(l-l^{\prime}\right)$ restricts the coupling to the cases in which the angular momentum quantum number of electron is increased by $\hbar m$ while that of nucleus correspondingly is decreased by $\hbar m$, or vice versa. Therefore it is meaningful to introduce the quantum number $q=k+l$, which is conserved by the vibronic coupling, and to note that the total Hamiltonian separates into blocks according to the value of $q$. The last term in Eq. (1) describes the matter-light interaction with dipole $\boldsymbol{\mu}=-r\left(\boldsymbol{\epsilon}_{x} \cos (\theta)+\boldsymbol{\epsilon}_{y} \sin (\theta)\right)$ (the nuclear contribution to the dipole can be safely neglected because the fields applied are not resonant with direct transitions in the nuclear coordinate) and electric field $\mathbf{E}(t)=\boldsymbol{\epsilon}_{x} E_{x}(t)+\boldsymbol{\epsilon}_{y} E_{y}(t)$ terms, where $\boldsymbol{\epsilon}_{u}$ is the unitary polarization vector pointing in the $u$-direction. The electric field of the laser pulses $E_{u}(t)$ is derived from the vector potential

$$
A_{u}(t)=\frac{E_{0}}{\omega} S(t) \sin \left(\omega t-\phi_{u}\right)
$$

as $E_{u}(t)=-\partial A_{u}(t) / \partial t$, where we use a sine-squared envelope function $S(t)=\tilde{\Theta}(t-\tau) \sin ^{2}\left(\frac{\pi t}{\tau}\right) . \quad E_{0}, \tau$ and $\omega$ are the maximum amplitude, pulse duration (start to end) and carrier frequency of the pulse, respectively, and $\tilde{\Theta}(t-\tau)$ is the inverse Heaviside step function. We take all pulse parameters for both $(x, y)$ polarization directions to be equal except for the $\phi_{x}$ and $\phi_{y}$ phases. A right circularly polarized pulse (RCP) corresponds to the phases $\left(\phi_{x}=\pi / 2, \phi_{y}=0\right)$ and a left circularly polarized pulse (LCP) to the phases $\left(\phi_{x}=0, \phi_{y}=\pi / 2\right)$.

\section{A. Ring currents under vibronic coupling effects}

The electronic component $J^{(\theta)}(\alpha, \theta, t)$ of the total wave function current at coordinates $(\alpha, \theta)$ follows from the continuity condition of the probability density ${ }^{35}$ and is given by

$$
J^{(\theta)}(\alpha, \theta, t)=\frac{1}{I_{\theta}} \operatorname{Re}\left\{\psi^{*}(\alpha, \theta, t) \hat{l}_{\theta} \psi(\alpha, \theta, t)\right\},
$$

where $\hat{l}_{\theta}=-i \hbar \partial_{\theta}$. The electronic component of the current as a function of the nuclear coordinate(s) is of not much practical use. In actual applications, one needs instead the electronic current averaged over the nuclear coordinates, which in the present model reads

$$
J_{e}(\theta, t)=\frac{1}{I_{\theta} 2 \pi} \int_{0}^{2 \pi} J^{(\theta)}(\alpha, \theta, t) d \alpha .
$$

Using the Born-Huang expansion, Eq. (5), with Eqs. (9) and (10), one arrives at the following expression for the nuclear-averaged electronic current: 


$$
J_{e}(\theta, t)=\frac{1}{I_{\theta} 2 \pi} \int_{0}^{2 \pi}\left[\sum_{l=-L}^{L} \hbar l\left|\chi_{l}(\alpha, t)\right|^{2}+\Re\left\{\sum_{l^{\prime}=-L}^{L} \sum_{l=-L}^{L}\left(1-\delta_{l^{\prime} l}\right) \hbar l \chi_{l^{\prime}}^{*}(\alpha, t) \chi_{l}(\alpha, t) e^{i\left(l-l^{\prime}\right) \theta}\right\}\right] d \alpha .
$$

The first term in the above equation is $\theta$-independent and describes the contribution of the $l$-th electronic state to the overall ring current. The second term is a fluctuating contribution originating from the interference between the $l$-th and $l^{\prime}$-th electronic basis states at a given nuclear geometry $\alpha$. This fluctuating component along $\theta$ vanishes when integrating $J_{e}(\theta, t)$ over the closed path of the electronic coordinate due to the imaginary exponential term. Thus, averaging over the nuclear coordinate and integrating over the electronic closed path one arrives at the expression for the net time-dependent electronic ring current

$$
J_{e}(t)=\frac{1}{4 \pi^{2} I_{\theta}} \int_{0}^{2 \pi} J_{e}(\theta, t) d \theta=\frac{\hbar}{4 \pi^{2} I_{\theta}} \sum_{l=-L}^{L} l P_{l}(t) .
$$

where $P_{l}(t)=\int_{0}^{2 \pi}\left|\chi_{l}(\alpha, t)\right|^{2} d \alpha$. This expression for the net ring current tells that the contribution of the $l$-th electronic-current state is proportional to the corresponding population, namely the nuclear coefficient squared in the $\mathrm{BH}$ wave function expansion. This simple expression is reached because our electronic diabatic basis consists, for convenience, of complex-valued current eigenstates instead of their real-valued linear combinations. In a molecular calculation one would introduce the corresponding $\varphi_{ \pm}^{E}$ diabatic electronic-current states to arrive at an analogous expression for the net ring currents.

Equation (12) illustrates how the creation of a stable imbalance of the population (the norm of the nuclear wavepackets) of the current components corresponding to opposite directions (i.e. $l= \pm 1$ in the model) results in an electronic ring current. If the population imbalance is stationary, so is the corresponding current. If, on the other hand, the electronic-current state populations vary, which can only be the result of either nonadiabatic couplings or interactions with an external electromagnetic field, the net electronic ring current $J_{e}(t)$ varies in time as well.

The coupling terms in Eq. (7) illustrate the basic mechanism of the electronic-nuclear interactions affecting ring currents, which consists of the exchange of angular momentum between the electron and the nucleus. According to Eq. (7) and restricting the electronic subspace to $l=\{-1,0,1\}$ as above, the basis state $|k, 0\rangle$ couples only to the states $|k+1,-1\rangle$ and $|k-1,+1\rangle$. The three basis states $|0,0\rangle,|1,-1\rangle$ and $|-1,1\rangle$ result in a $3 \times 3$ Hamiltonian matrix with $q=0$ that upon diagonalization yields three non-degenerate vibronic eigenstates (eigenstates of the complete nuclear-electronic Hamiltonian)

$$
\left|j_{0}\right\rangle=c_{-1,1}^{j_{0}}|-1,1\rangle+c_{0,0}^{j_{0}}|0,0\rangle+c_{1,-1}^{j_{0}}|1,-1\rangle,
$$

where the notation $\left|j_{q}\right\rangle$ is used for the vibronic states.
The expansion coefficients obey $\left|c_{-1,1}^{j_{0}}\right|=\left|c_{1,-1}^{j_{0}}\right|$ on symmetry grounds. Therefore, not surprisingly vibronic eigenstates with $q=0$ feature no net electronic current. The operation of reversing the sense of circulation of all particles, $(k \rightarrow-k, l \rightarrow-l)$, is closed within the basis states, and the corresponding eigenstates belong to a completely symmetric representation.

On the other hand, the doubly degenerate vibronic states originate from the two blocks with quantum numbers $\pm q$ and $|q| \geq 1$. One such block, e.g., $|k, 0\rangle$, $|k+1,-1\rangle$ and $|k-1,+1\rangle$ with $q=1$, results in the corresponding $3 \times 3$ Hamiltonian matrix which can be diagonalized to yield the three non-degenerate states

$$
\left|j_{1}\right\rangle=c_{k-1,1}^{j_{1}}|k-1,1\rangle+c_{k, 0}^{j_{1}}|k, 0\rangle+c_{k+1,-1}^{j_{1}}|k+1,-1\rangle .
$$

These vibronic eigenstates feature an electronic ring current because, in general, $\left|c_{k-1,1}^{j_{q}}\right| \neq\left|c_{k+1,-1}^{j_{q}}\right|$ and therefore they support an imbalance of the contribution to each electronic current circulation direction.

Inverting the sense of circulation of all particles, $q \rightarrow$ $-q$, results in the basis functions $|-k, 0\rangle,|-k+1,-1\rangle$ and $|-k-1,+1\rangle$ with $q=-1$. The diagonalization of the corresponding Hamiltonian matrix yields the triad of $\left|j_{-1}\right\rangle$ states, degenerate one to one with the corresponding $\left|j_{1}\right\rangle$ eigenstates. Finally, the expansion coefficients of the vibronic eigenstates $\left|j_{1}\right\rangle$ and $\left|j_{-1}\right\rangle$ are related by $c_{k \pm 1, \mp 1}^{j_{1}}=c_{-k \mp 1, \pm 1}^{j_{-1}}$ and $c_{k, 0}^{j_{1}}=c_{-k, 0}^{j_{-1}}$.

We can already see through the general strategy to generate a ring current under nonadiabatic electronicnuclear couplings. Stated in terms of eigenstates of the complete Hamiltonian, it will consist in creating a population imbalance within pairs of vibronic (not electronic) states $\left|j_{q}\right\rangle$ and $\left|j_{-q}\right\rangle$. If each $\left(j_{q}, j_{-q}\right)$ pair of eigenstates of a given $\pm q$ is equally populated, no net current will be achieved. This is because the individual net currents of $j_{q}$ and $j_{-q}$ are equal and opposite, as $c_{k \pm 1, \mp 1}^{j_{q}}=c_{-k \mp 1, \pm 1}^{j_{-q}}$.

\section{RESULTS AND DISCUSSION}

Let us assume that a narrow-band laser pulse resonant with the transition between the absolute ground state $\left|0_{0}\right\rangle$ and $\left|j_{ \pm q}\right\rangle$, i.e. with central frequency $\omega=\left(E_{j_{q}}-E_{0}\right) / \hbar$, interacts with the system in its ground state and transfers population to this pair of eigenstates only. In this case, the vibronic wavepacket after the pulse is

$$
|\psi(t)\rangle=A_{0}(t)\left|0_{0}\right\rangle+A_{j_{q}}(t)\left|j_{q}\right\rangle+A_{j_{-q}}(t)\left|j_{-q}\right\rangle,
$$

where $\left|A_{0}(t)\right|^{2}$ and $\left|A_{j_{ \pm q}}(t)\right|^{2}$ are the populations of the ground and excited vibronic states. For a linearly polar- 
ized pulse $\left|A_{j_{q}}(t)\right|^{2}=\left|A_{j_{-q}}(t)\right|^{2}$ follows, whereas a circularly polarized pulse results in a complete imbalance with one of the two components receiving zero population. This fact can easily be checked analytically and it will be illustrated in the numerical results below. We can further note that the dipole operator in Eq. (1) changes the quantum number of the electron by \pm 1 , and that the ground state belongs to the $q=0$ block. Hence, for weak, perturbative pulses interacting with the ground state the currents are restricted to the subspaces with $q= \pm 1$. The discussion focuses on this one-photon limit case in the following.

The question we ask is, what are the fundamental characteristics of the ring currents that are generated by such narrow-band pulses? Using that $P_{l}(t)=|\langle l \mid \psi(t)\rangle|^{2}$ in Eq. (12) and assuming that the system is described by the state in Eq. (15) one arrives at the ring current associated with a pair of vibronic states

$$
J_{j_{1}}(t)=\frac{\hbar}{4 \pi^{2} I_{\theta}}\left(\left|A_{j_{1}}(t)\right|^{2}-\left|A_{j_{-1}}(t)\right|^{2}\right) Y_{j_{1}}
$$

where $Y_{j_{1}}=\left|c_{0,1}^{j_{1}}\right|^{2}-\left|c_{2,-1}^{j_{1}}\right|^{2}$ and we have used the relation $Y_{j_{1}}=-Y_{j_{-1}}$ to separate the common factor. The state-dependent molecular factor $Y_{j_{1}}$ can take values in the range $(-1,1)$ and it corresponds to the imbalance of positive versus negative electronic current of the $\left|j_{1}\right\rangle$ vibronic eigenstate. Its value is uniquely determined by the strength of the vibronic coupling of the system. In the complete absence of coupling, right- and left-rotating electronic contributions do not contribute simultaneously to the same degenerate eigenstate because they are not connected via the vibronic coupling terms, Eq. (7), in the Hamiltonian. In this case, $Y_{j_{1}}= \pm 1$. For strong nonadiabatic coupling $Y_{j_{1}} \rightarrow 0$ and only a small current within the $j_{1}$ subspace is possible. Summarizing, the generation of a ring current within the degenerate space of two vibronic states of the complete Hamiltonian requires two ingredients: that the molecular factor $Y_{j_{q}}$ is significant for the $j_{ \pm q}$ states, and that an imbalance of the populations $\left|A_{j_{q}}(t)\right|^{2}$ and $\left|A_{j_{-}}(t)\right|^{2}$ is achieved by the circularly polarized nature of the light interacting with the system. Since, by assumption, only two eigenstates with energy $E_{j_{1}}$ are involved, such a current will be stationary (cf. Eq. (16)) once the laser pulse is over. Let us consider now the case that the incoming pulse is short and has sufficient bandwidth to overlap with transitions to a group of optically bright vibronic eigenstates. In this case, starting again from Eq. (12) and assuming that after the laser pulse the wave function consists of a linear superposition of all states in the $q \pm 1$ blocks, one arrives at a generalization of Eq. (16),

$$
J_{e}(t)=\frac{\hbar}{4 \pi^{2} I_{\theta}}\left[\sum_{j_{q}}\left(\left|A_{j_{q}}(t)\right|^{2}-\left|A_{j_{-q}}\right|^{2}\right) Y_{j_{q}}+\left(2 \Re\left\{A_{j_{q}}^{*}(t) A_{j_{q}^{\prime}}(t)\right\}-2 \Re\left\{A_{j_{-q}}^{*}(t) A_{j_{-q}^{\prime}}(t)\right\}\right) Y_{j_{q} j_{q}^{\prime}}\right] .
$$

A derivation of the most general expression without the limitation to the $q= \pm 1$ blocks is given in the supporting information (SI). After the pulse is over, oscillatory contributions to the ring current arise from interferences within each $q= \pm 1$ branch in the second term of the expression. Clearly, no quantum interference between the different $q$ blocks resulting in current oscillations takes place. Moreover, a linearly polarized pulse creates the same populations and phases in the two $q$ blocks and the terms in parenthesis vanish in the same as discussed for the narrow-band pulse. A short, circularly polarized pulse, instead, creates a superposition of states of either the $q=1$ or $q=-1$ branches, resulting in an oscillatory ring current. The molecular factor in the second term is now $Y_{j_{q} j_{q}^{\prime}}=\left(c_{0,1}^{j_{q}}\right)^{*} c_{0,1}^{j_{q}^{\prime}}-\left(c_{2,-1}^{j_{q}}\right)^{*} c_{2,-1}^{j_{q}^{\prime}}$, the generalization to two states of the product of the coefficients for each current direction.

In order to generate net electronic currents according to Eq. (17), the task is to identify and target specific degenerate vibronic eigenstates that are characterized by a net imbalance of the electronic current in each direction. For molecules with a $C_{3}$ axis, this means to identify bright degenerate vibronic states $\left|j_{ \pm}\right\rangle$for which the molecular factor $Y_{j}=\left|\left\langle E_{+} \mid j_{+}\right\rangle\right|^{2}-\left|\left\langle E_{-} \mid j_{+}\right\rangle\right|^{2}$ is as close to 1 (or -1) as possible. Here the $E_{ \pm}$diabatic electronic states play exactly the same role as the $l= \pm 1$ electronic states in the RRM.

Figure 1 provides the $l=\{-1,0,1\}$ contributions for the ground state and first eight excited states $\left|j_{q}\right\rangle$. For degenerate states, only the $q=(1,2)$ branches are shown. The molecular factor for each state follows from subtracting the right (purple) from the left (magenta) columns. Only the states $0_{ \pm 1}, 1_{ \pm 1}$ and $2_{ \pm 1}$ have a non-zero transition-dipole moment $\left(\left|\mu_{0_{0} j_{q}}\right|^{2}\right)$ with the $0_{0}$ ground state. States $1_{1}$ and $2_{1}$ seem both good candidates to generate a ring current by applying a $\mathrm{RCP}$ pulse. Recall that RCP and LCP pulses couple the ground state to the $q=+1$ and $q=-1$ subspaces, respectively. The molecular factor for $1_{1}$ is about 0.4 for the considered model parameters, whereas it is about -0.52 for the $2_{1}$ state. This results in the counterintuitive fact that a RCP resonant with the $0_{0} \rightarrow 2_{1}$ transition creates a left-circulating electronic current. This is a consequence of the electronic-nuclear coupling. The basis state $|2,-1\rangle$ has a larger weight than the basis state $|0,1\rangle$ in the vibronic state $\left|2_{1}\right\rangle$. However, this 


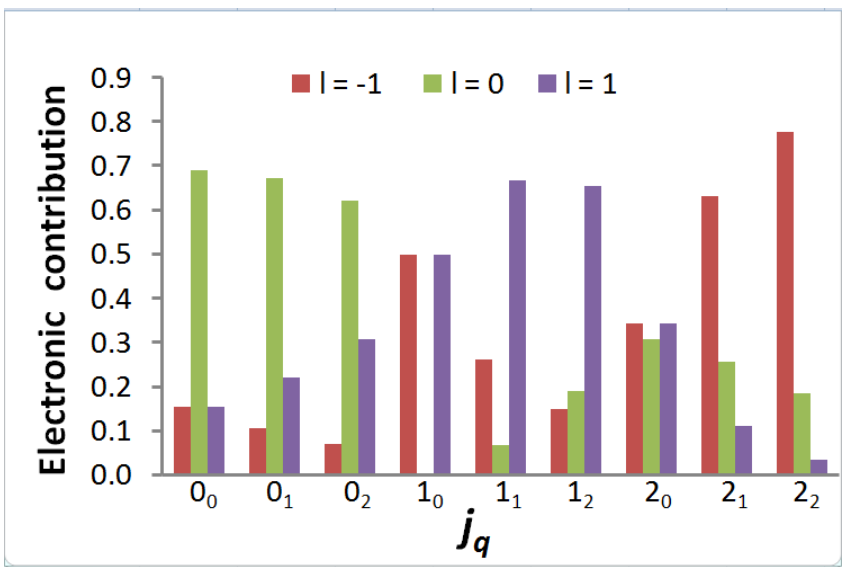

FIG. 1. Contributions of the electronic ground $(l=0)$ and the electronic excited $(l=-1$ and $l=1)$ states for the first few vibronic states. The transition dipole moment $\left|\mu_{0_{0} j_{q}}\right|^{2}$ is non-vanishing for $j_{q}=0_{1}, 1_{1}$ and $2_{1}$

transition has a small Franck-Condon factor because the main basis contributions to each eigenstate cancel: $\left\langle 0_{0}\left|\mu_{e}\right| 2_{1}\right\rangle \approx\left\langle 0,0\left|\mu_{e}\right| 2,-1\right\rangle=\langle 0 \mid 2\rangle\left\langle 0\left|\mu_{e}\right|-1\right\rangle=0$. Therefore, in what follows, we study the situation in which a $\mathrm{RCP}$ laser pulse is resonant with the $0_{0} \rightarrow 1_{1}$ transition.

Figure 2 shows the current generated by pulses of various durations, ranging from 50 to 600 fs. Instead of the net current, we plot the quantity $\tilde{J}_{e}(t)=J_{e}(t) / \gamma(1-$ $\left.P_{0}(\tau)\right)$, where $\gamma=\hbar / 4 \pi^{2} I_{\theta}$ and $P_{0}(\tau)$ is the population of the ground state after the pulse is over. This normalized net current can take values between $(-1,1)$ and its introduction serves two purposes. First, the normalization by the amount of excitation induced by the laser makes the results independent of the laser intensity in the perturbative regime. We have lowered the laser intensity to an amount in which the normalized current becomes independent of the laser intensity. Second, it pinpoints the fact that not all the population transferred to a vibronic eigenstate contributes to the net current. This is limited by the molecular factor. For example, a molecular factor of 0.4 sets the limit of the normalized current associated with this eigenstate to 0.4 . The effect of the electronicnuclear coupling is studied by multiplying the coupling constants in Eq. (4) by a factor $\lambda$ in the $[0,1]$ range. A long RCP in the full coupling case, Fig. 2(c), populates exclusively the $1_{1}$ state. It results in a stationary current $\tilde{J}_{e}(t)$ limited to about 0.4 after the pulse, which exactly coincides with the $Y_{1_{1}}$ value. An admixture of the $1_{-1}$ state would reduce the amount of current with respect to this theoretical maximum. Thus, as expected, the RCP pulse couples exclusively to the $q=+1$ subspace and achieves the maximum ring current permitted for the $0_{0} \rightarrow 1_{1}$ transition. As the pulse becomes shorter, a superposition of the $0_{1}, 1_{1}$ and $2_{1}$ states is obtained instead, leading to an oscillatory current. The oscillatory current generated at the full coupling limit (cf. Fig. 2(c)) reveals changes in the direction of the ring currents
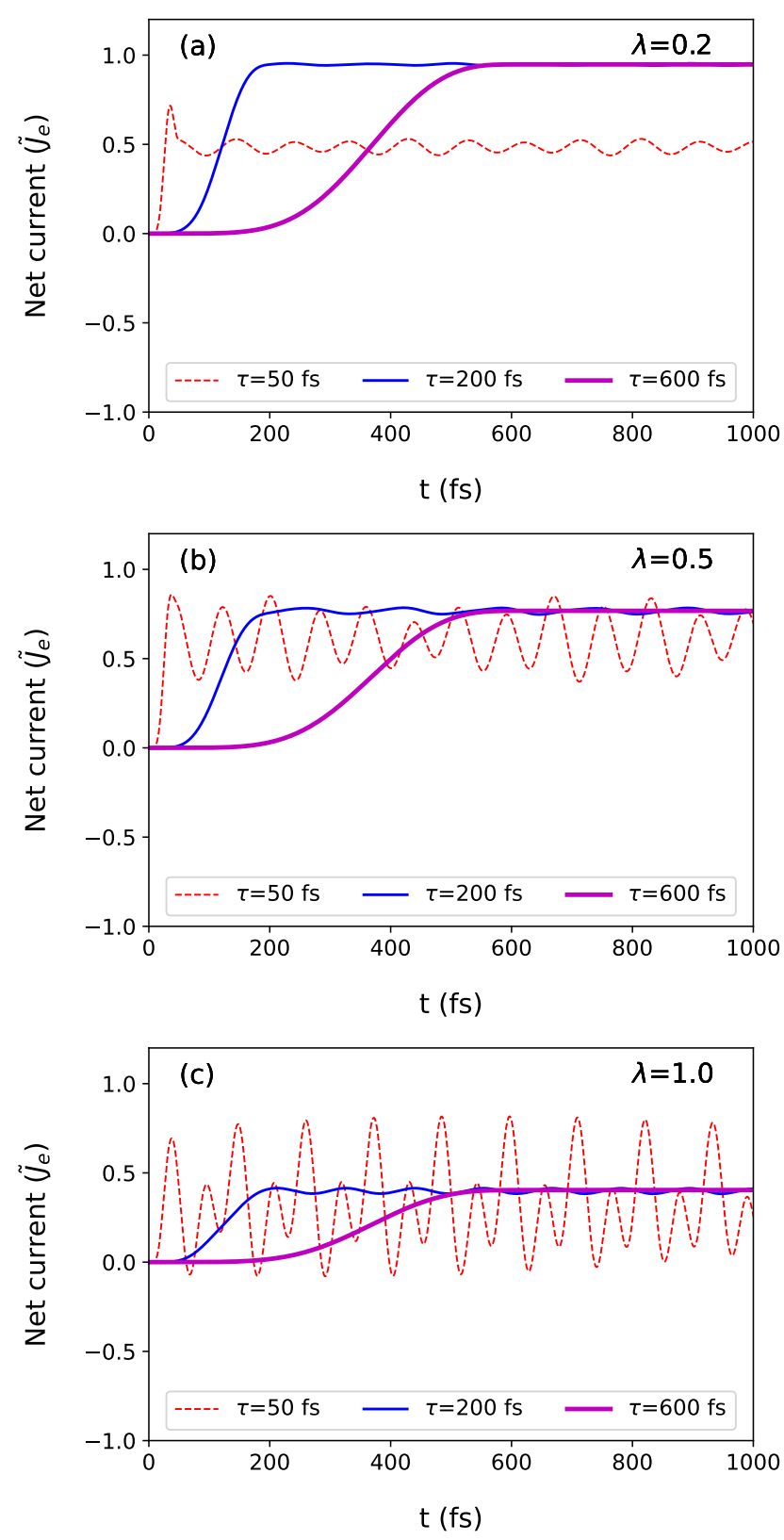

FIG. 2. The normalized net currents $\left(\tilde{J}_{e}\right)$ at coupling strengths $\lambda=0.2$ (a), $\lambda=0.5$ (b) and $\lambda=1.0$ (c), generated by the action of RCP with different durations $(\tau)$, as function of time.

in time, which originate from the joint contribution of the basis states $|0,1\rangle$ and $|2,-1\rangle$ to the vibronic states $1_{1}$ and $2_{1}$. Going back to the equivalent picture of the $\chi_{l}(\alpha, t)$ nuclear wavepackets in the $\mathrm{BH}$ representation of the wave function, the coupling terms in the VCH Hamiltonian (6) couple the various $l$ contributions and result in the current oscillations, in which the nucleus and the electron exchange angular momentum.

As the electronic-nuclear coupling diminishes for $\lambda<1$ two effects are seen (cf. Fig. 2(a-b)). First, the normalized net current after the pulse increases and ap- 

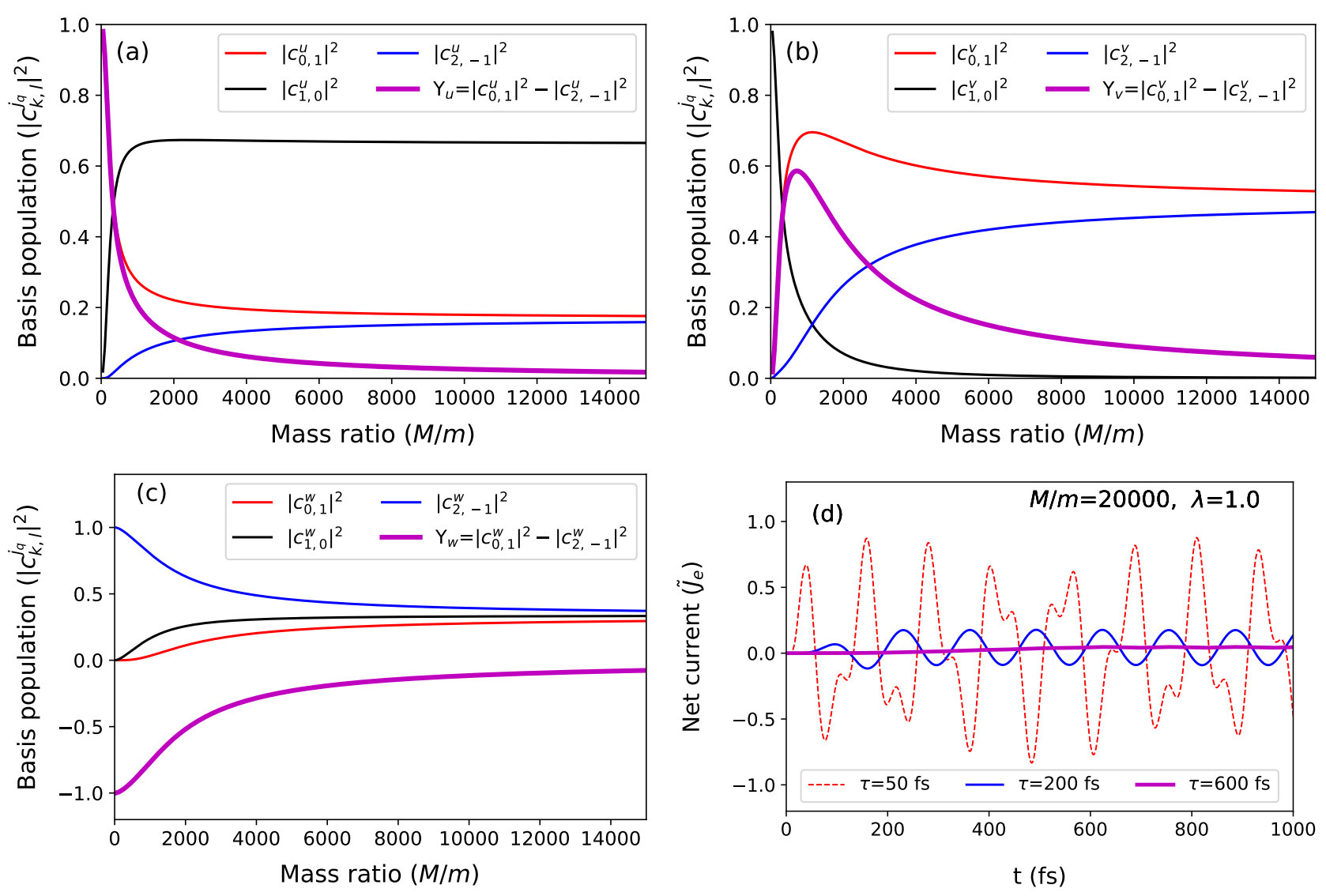

FIG. 3. The populations of $|k, l\rangle$ basis consisting of $|0,1\rangle,|1,0\rangle$ and $|2,-1\rangle$ states in the BH expansion of the three vibronic states $j_{1}=u(\mathrm{a}), v(\mathrm{~b})$ and $w(\mathrm{c})$ as function of nuclear electron mass ratio $(M / m)$, at the full coupling limit(i.e. $\left.\lambda=1.0\right)$. In $(\mathrm{d})$, the stationary and oscillatory currents generated by the action of RCP with different durations $(\tau)$, as function of time, are shown for $M / m=20000$ at $\lambda=1.0$.

proaches a value of 1 . With a reduced vibronic coupling, the right-rotating component in the $1_{1}$ vibronic state becomes more dominant, meaning that the molecular factor approaches 1 as well. Second, the current generated by the shortest pulse becomes less oscillatory and for $\lambda=0$ (not shown) it does not oscillate at all. Even if the pulse has enough bandwith to overlap with various states of the $q=1$ branch, only the one with the largest $|0,1\rangle$ contribution has a significant transition dipole. Other vibronic states in this branch, in contrast, have a negligible Franck-Condon factor with the ground state as the vibronic coupling is reduced.

Finally, we approach the question of the nature of the generated currents from the point of view of the mass ratio $M / m$ between the nucleus and the electron, which in the previous calculations was always set to 2000. As the nuclear mass increases, the contribution to each electronic circulation direction in each vibronic eigenstate $\left(0_{1}, 1_{1}, 2_{1}\right)$ of the $q=1$ block, shown by red and blue curves in Fig. 3, tends to become equal, which brings the molecular factor, shown in magenta, towards zero in the $M / m \rightarrow \infty$ limit. Essentially, the basis states $|2,-1\rangle$ and $|0,1\rangle$ become energetically degenerate and contribute equally to the vibronic eigenstates. As we discussed before, a RCP/LCP only permits transitions into either the $q=1$ or $q=-1$ branch, respectively, so a stationary ring current is not possible in this limit (see magenta line in Fig. 3(d)). Extending the argument to the usual $E \times e$ molecular Jahn-Teller scenario, i.e. with direct coupling between electronic states of $E$ symmetry, this corresponds to the situation where the nuclear wave function localizes at the bottom of the Mexican hat potential (see e.g. Ref. 36, Ch. 10) and has a negligible amplitude at the point in configuration space where the diabatic electronic states are degenerate. In this BO limit, no stationary ring currents can be generated by a laser interacting with the ground state. The $\mathrm{BO}$ limit should not be confused with fixing the nuclei at a specific geometry. Note that in the RRM and in the limit $M / m \rightarrow \infty$ the nucleus is still completely delocalized along its coordinate $\alpha$.

Even though stationary ring currents are not possible in the $M / m \rightarrow \infty$, the generation of an oscillatory ring current remains possible using a short RCP/LCP pulse of sufficient coherent bandwidth to excite simultaneously the various states in the corresponding $q= \pm 1$ subspace, 
as can be seen in Eq. (17). The currents are shown in Fig. 3(d) in red and blue curves. The vibronic eigenstates do not support a ring current individually, but the offdiagonal molecular factors $Y_{j_{1}, j_{1}^{\prime}}$ in Eq. (17) are not zero, as can be seen by inspecting the different values of the coefficients for the same basis state in Fig. 3(a-c). All vibronic states with $q=1$ have some non-zero contribution of the basis state $|0,1\rangle$ and therefore all transitions $0_{0} \rightarrow j_{1}$ are allowed. However, the time-averaged ring current vanishes (see Fig. 3(d)) because each vibronic state in the linear superposition carries the same weight for both electronic circulation directions. Hence, in this $\mathrm{BO}$ limit, it is not possible to permanently favor one circulation direction over the other. This may not be as dramatic as it sounds, as the mass ratio between nuclei and electrons is not infinite, and because the vibronic coupling is not infinitely strong either, thus in general allowing for the existence of vibronic states in the $q= \pm 1$ subspaces ( $E_{ \pm}$vibronic states in molecules) that favor to some extent a specific circulation direction of the electrons.

\section{CONCLUSIONS}

We have analyzed in detail the generation of stationary and oscillatory ring currents in a model system featuring the necessary symmetry conditions to support ring currents that are required in more complex systems as well as a transparent vibronic coupling mechanism. In the spirit of the Moshinsky-Kittel ${ }^{37}$, the ShinMetiu $^{38}$ or Loguet-Higgins models ${ }^{30}$, the RRM Hamiltonian allows for both analytical considerations and exact numerical solutions, both within and beyond the BO approximation. Electron-nuclear interactions hinder the maximum amount of stationary ring current that can be achieved by applying long, circularly polarized pulses. We characterize this intrinsic limitation to the maximum ring current through state-dependent molecular factors that correspond to the difference between right- and left-circulation contributions of the electrons in a specific vibronic state. These molecular factors depend on the strength of the nonadiabatic coupling between electrons and nuclei. Short, circularly polarized pulses with sufficient bandwidth lead to oscillatory currents created by the superposition of vibronic eigenstates within the same symmetry block of the complete Hamiltonian. In the limit of a large nuclear-electronic mass ratio, where the $\mathrm{BO}$ approximation is fulfilled, degenerate pairs of vibronic eigenstates cannot support stationary currents and the oscillatory currents generated by RCP/LCP broad-band pulses average out to zero over time. The observations made on the RRM system are general and have consequences for applications of lasergenerated ring currents in molecules and materials.

\section{SUPPORTING MATERIAL}

See the SI for the derivation of the general expression for the oscillatory currents.

\section{DATA AVAILABLE IN ARTICLE OR SI}

The data that supports the findings of this study are available within the article [and its SI].

\section{ACKNOWLEDGEMENT}

The authors thank Prof. Wolfgang Domcke for insightful comments on an earlier version of the manuscript and Prof. Horst Köppel for helpful discussions. The authors declare no conflicts of interest.

${ }^{1}$ I. Barth, J. Manz, Y. Shigeta, and K. Yagi, J. Am. Chem. Soc. 128, 7043 (2006).

${ }^{2}$ I. Barth and J. Manz, Angew. Chem. Int. Ed. 45, 2962 (2006).

${ }^{3}$ I. Barth and J. Manz, Phys. Rev. A 75, 012510 (2007).

${ }^{4}$ I. Barth and J. Manz, in Progress in Ultrafast Intense Laser Science VI (Springer, 2010) pp. 21-44.

${ }^{5}$ I. S. Ulusoy and M. Nest, J. Am. Chem. Soc. 133, 20230 (2011).

${ }^{6}$ H. Mineo, M. Yamaki, G.-S. Kim, Y. Teranishi, S. H. Lin, and Y. Fujimura, Phys. Chem. Chem. Phys. 18, 26786 (2016).

${ }^{7}$ H. Mineo and Y. Fujimura, J. Phys. Chem. Lett. 8, 2019 (2017).

${ }^{8}$ H. Mineo and Y. Fujimura, J. Chem. Phys. 147, 224301 (2017).

${ }^{9}$ C. Liu, J. Manz, K. Ohmori, C. Sommer, N. Takei, J. C. Tremblay, and Y. Zhang, Phys. Rev. Lett. 121, 173201 (2018).

${ }^{10}$ M. Kanno, H. Kono, and Y. Fujimura, Appl. Sci. 8, 2347 (2018).

${ }^{11}$ Y. V. Pershin and C. Piermarocchi, Phys. Rev. B 72, 245331 (2005).

${ }^{12}$ A. Matos-Abiague and J. Berakdar, Phys. Rev. Lett. 94, 166801 (2005).

${ }^{13}$ E. Räsänen, A. Castro, J. Werschnik, A. Rubio, and E. K. U. Gross, Phys. Rev. Lett. 98, 157404 (2007).

14 J. E. Anthony, Chem. Rev. 106, 5028 (2006).

${ }^{15}$ A. D. Bandrauk, S. Chelkowski, and H. S. Nguyen, Int. J. Quantum Chem. 100, 834 (2004).

${ }^{16}$ F. Krausz and M. Ivanov, Rev. Mod. Phys. 81, 163 (2009).

${ }^{17}$ K.-J. Yuan, C.-C. Shu, D. Dong, and A. D. Bandrauk, J. Phys. Chem. Lett. 8, 2229 (2017).

${ }^{18}$ A. I. Kuleff, J. Breidbach, and L. S. Cederbaum, J. Chem. Phys. 123, 044111 (2005).

${ }^{19}$ F. Remacle and R. D. Levine, Proc. Nat. Acad. Sci. 103, 6793 (2006).

${ }^{20}$ P. M. Kraus, B. Mignolet, D. Baykusheva, A. Rupenyan, L. Horný, E. F. Penka, G. Grassi, O. I. Tolstikhin, J. Schneider, F. Jensen, L. B. Madsen, A. D. Bandrauk, F. Remacle, and H. J. Wörner, Science 350, 790 (2015).

${ }^{21}$ F. Calegari, D. Ayuso, A. Trabattoni, L. Belshaw, S. D. Camillis, S. Anumula, F. Frassetto, L. Poletto, A. Palacios, P. Decleva, J. B. Greenwood, F. Martin, and M. Nisoli, Science 346, 336 (2014)

${ }^{22}$ M. Vacher, M. J. Bearpark, M. A. Robb, and J. P. Malhado, Phys. Rev. Lett. 118, 083001 (2017).

${ }^{23}$ C. Arnold, O. Vendrell, and R. Santra, Phys. Rev. A 95, 033425 (2017).

${ }^{24}$ H. Timmers, Z. Li, N. Shivaram, R. Santra, O. Vendrell, and A. Sandhu, Phys. Rev. Lett. 113, 113003 (2014).

${ }^{25}$ V. Despré, A. Marciniak, V. Loriot, M. C. E. Galbraith, A. Rouzée, M. J. J. Vrakking, F. Lépine, and A. I. Kuleff, J. Phys. Chem. Lett. 6, 426 (2015). 
${ }^{26}$ Z. Li, M. E.-A. Madjet, O. Vendrell, and R. Santra, Phys. Rev. Lett. 110, 038302 (2013).

${ }^{27}$ M. Kanno, H. Kono, Y. Fujimura, and S. H. Lin, Phys. Rev. Lett. 104, 108302 (2010).

${ }^{28}$ M. Kanno, Y. Ono, H. Kono, and Y. Fujimura, J. Phys. Chem. A 116, 11260 (2012).

${ }^{29}$ W. D. H. Köppel and L. Cederbaum, J. Chem. Phys. 74, 2945 (1981).

${ }^{30}$ H. C. Longuet-Higgins, U. Öpik, M. H. L. Pryce, and R. A. Sack, Proc. R. Soc. A 244, 1 (1958).

${ }^{31}$ H. Köppel, W. Domcke, and L. Cederbaum, Adv. Chem. Phys. $\mathbf{5 7}, 59$ (1984).

${ }^{32} \mathrm{M}$. Shapiro and P. Brumer, Principles of the quantum control of molecular processes (Wiley-VCH, 2003).

${ }^{33} \mathrm{M}$. Born and K. Huang, Dynamical theory of crystal lattices (Clarendon press, 1954).

${ }^{34}$ I. B. Bersuker, The Jahn-Teller effect (Cambridge University Press, 2006).

${ }^{35}$ A. Messiah, Quantum mechanics (Dover Publications, Mineola, N.Y, 1999).

${ }^{36}$ W. Domcke, D. Yarkony, et al., Conical intersections: electronic structure, dynamics \& spectroscopy, Vol. 15 (World Scientific, 2004).

${ }^{37}$ M. Moshinsky and C. Kittel, Proc. Nat. Acad. Sci. 60, 1110 (1968).

${ }^{38}$ S. Shin and H. Metiu, J. Chem. Phys. 102, 9285 (1995). 